\title{
Fundamental Study on a Tracer Gas Experimental Method that uses Dynamic Steady State Concentration and can be Applied to an Air Recirculating System
}

\author{
Naoya Ikemura ${ }^{1 *}$, Takashi Kurabuchi ${ }^{1}$, Jinya Takeuchi ${ }^{2}$, Hazime Yoshino ${ }^{3}$ and Yoshihiro Toriumi ${ }^{4}$ \\ ${ }^{1}$ Tokyo University of Science, Tokyo, Japan \\ ${ }^{2}$ Akita Prefectural University, Akita, Japan \\ ${ }^{3}$ Nippon Institute of Technology, Saitama, Japan \\ ${ }^{4}$ Tokyo Denki University, Saitama, Japan
}

\begin{abstract}
In the previous paper, theoretical consideration of the tracer gas experimental method that uses dynamic steady state concentration was made. In this study, the feasibility of the tracer gas experimental method was examined based on a laboratory experiment. It was confirmed that a dynamic steady state concentration in case of negative generation of tracer gas corresponds to the age of air in an open-air system. The sum of dynamic steady state concentrations in case of positive generation of tracer gas and that for negative generation in the air recirculating part of a room is similar to the steady state concentration in an open air system.
\end{abstract}

\section{Introduction}

Tracer gas experimental methods have been widely used to evaluate the ventilation effectiveness or gaseous contaminant removal efficiency of a space. In the case of multi air conditioners within buildings, air purifiers, etc., the temperature, humidity, and cleanliness of the air change during the course of passing through the apparatus. It is desirable to be able to quantitatively evaluate the efficiency of improvements concerning indoor environments.

However, when tracer gas is supplied under non-ventilated conditions, the concentration rises endlessly, so no suitable experimental method has been established. Such a system without ventilation is called an air recirculating system. In the previous study $^{1}$, we proposed a theory that enables a tracer gas experiment on the air recirculating system by using the concept of the dynamic steady-state concentration established when supplying tracer gas.

In this study, we applied the same theory to laboratory experiments and examined the possibility of tracer gas in the air recirculating system.

\section{Outline of dynamic steady-state concentration}

\subsection{Concept of dynamic steady-state concentration}

\footnotetext{
${ }^{*}$ Naoya Ikemura: kuro.ground6.gmail.com
}

When there is a positive source in an air recirculating system room without leakage and a negative source in the air recirculating part, the concentration rise is cancelled as shown in Figure 1. If we consider the problem of concentration diffusion as a passive scalar transport problem, we find that because the phenomenon is linear, it is possible to synthesize each concentration distribution. The sum of each concentration distribution represents a stationary concentration distribution with no time change.

We define the dynamic steady-state concentration as follows, each time-varying concentration constituting the steady concentration distribution.

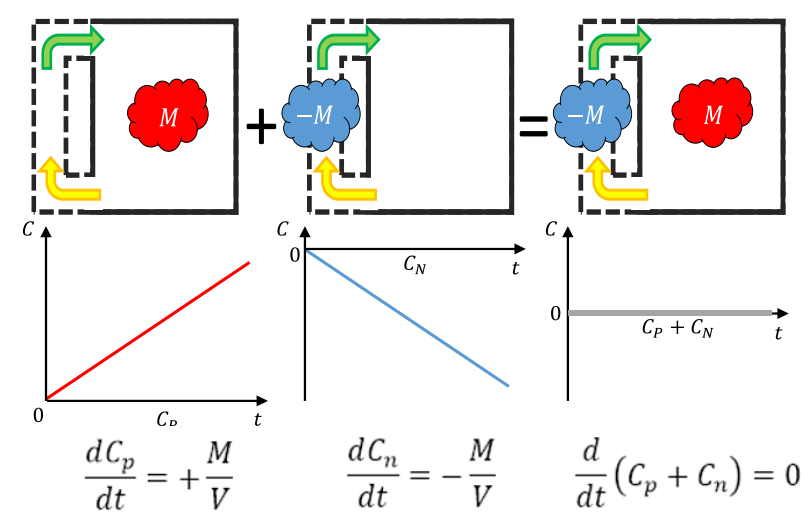

Fig. 1. Dynamic steady-state concentrations in a room with complete mixing conditions 


\subsection{Relationship between age of air and dynamic steady-state concentration}

The steady-state concentration distribution in a room with an air open system is the sum of the dynamic steady-state concentrations in an equal sized room with an air recirculating system. As can be seen in Figure 2, equations (1) and (2) point out concentration changes at a constant rate. This means that the sum of intercepts is a steady-state concentration. Also, considering the problem of concentration transport in the case of a circulating negative source as the advection problem in the block model, Equation (3) can be formulated. Substituting Equation (2) into Equation (3) yields Equation (4). This is an expression starting the steady concentration when there is a uniform source per unit volume.

Therefore, the dynamic steady state concentration in the case of a single source in an air recirculating part of the room represents the age of air.

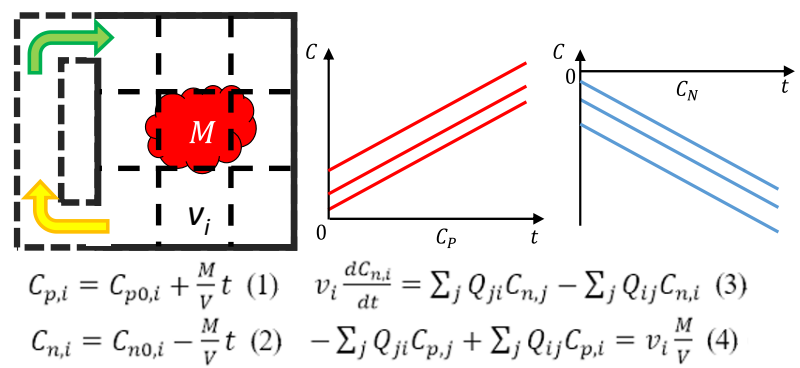

Fig. 2. Dynamic steady-state concentrations in the room with incomplete mixing conditions

\section{Outline of experiment}

\subsection{Experimental Verification}

Figure 3 shows an outline of the laboratory performing the tracer gas experiment. The experiment was conducted in a $3.5 \times 3.5 \times 2.8 \mathrm{~m}$ laboratory with a switchable ventilation system. This type of ventilation system can switch between the open system, which takes in fresh outside air and exhausts indoor air to the outside, and the air recirculating system where supply and exhaust are coupled in the air recirculating section of room.

A gas analyzer using an active method and a data logger using a passive method were used to measure the gas. the experiment was measured in consideration of the equipment difference ${ }^{2}$. A total of $24 \mathrm{CO}_{2}$ data loggers were installed in the vertical section of the room to measure concentration distributions and their changes with time. The supply inlet (SI) and exhaust outlet (EO) of the ventilation system and the room sources were installed in the same section. The generation method is the following four cases. In the EO side occurrence, two cases of generation were found in the vicinity of the same EO, regardless of whether a blower for stirring the tracer gas was used or not; in the SI side occurrence, two cases were found in the

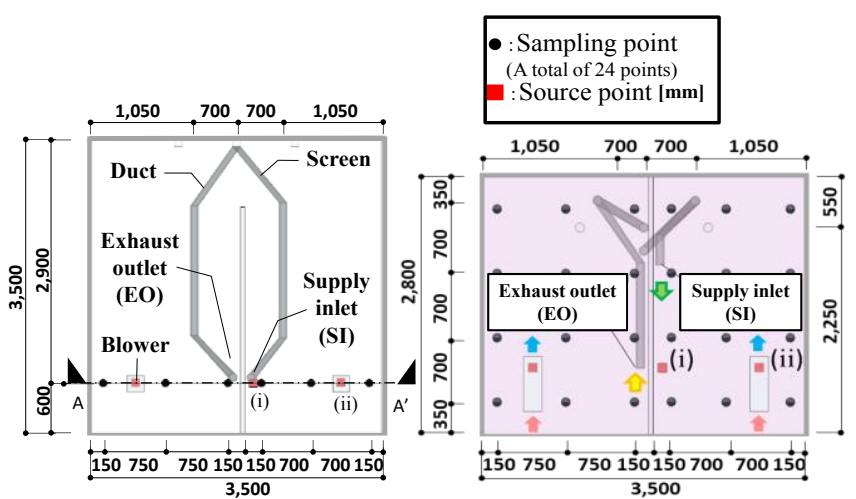

(a) Floor Plan

(b) Cross Screen

Fig. 3. Outline of the laboratory vicinity of the SI (i) in the absence of a blower and (ii) where there is a blower. Suction and discharge concentrations were measured with a gas analyzer, and tracer gas was generated quantitatively by $\mathrm{CO} 2$ mass flow.

\subsection{Experimental cases}

Table 1 shows the experimental cases, explaining the case of an open-air system. Case A is the age of air distributed by both the step-up and step-down methods. Case B and $\mathrm{C}$ are the ages of air distributed where blowers were installed in the vicinity of an EO and an SI, respectively. Cases D and F are those in which point sources were generated in the vicinity of an EO and of an SI, respectively. In addition, cases E and $G$ generated each source inside the blower in the vicinity of an EO and of an SI. For the open system, we conducted experiments on these seven cases.

In describing the case of the air recirculating system, Case AR is where the source was generated in the air recirculating part. Cases $\mathrm{BR}$ and $\mathrm{CR}$ are where a source was generated in the air recirculating part when a blower was installed in the vicinity of an EO and of an SI, respectively. Cases DR, ER, FR and GR are where point sources were generated in the same place as cases D, E, F and G, respectively. Experiments were also conducted on these seven cases for the air recirculating system.

\subsection{Results of the experiment}

Figure 4 shows the concentration transition by tracer gas experiment using dynamic steady-state concentration. In the experiment, we considered device differences and response times of the measuring equipment.

Measurement concentration is dimensionless and is determined using the ratio between the generation amount and the ventilation amount (M/Q). In the air recirculating system, it was impossible to conduct a tracer gas experiment to generate a negative source in the air recirculating system. Therefore, in the experiment, the sign of the concentration was reversed after determining the positive source.

Figure 4 (a) and (b) show that the concentration rises at a significantly constant rate. Figure 4 (c) shows the 


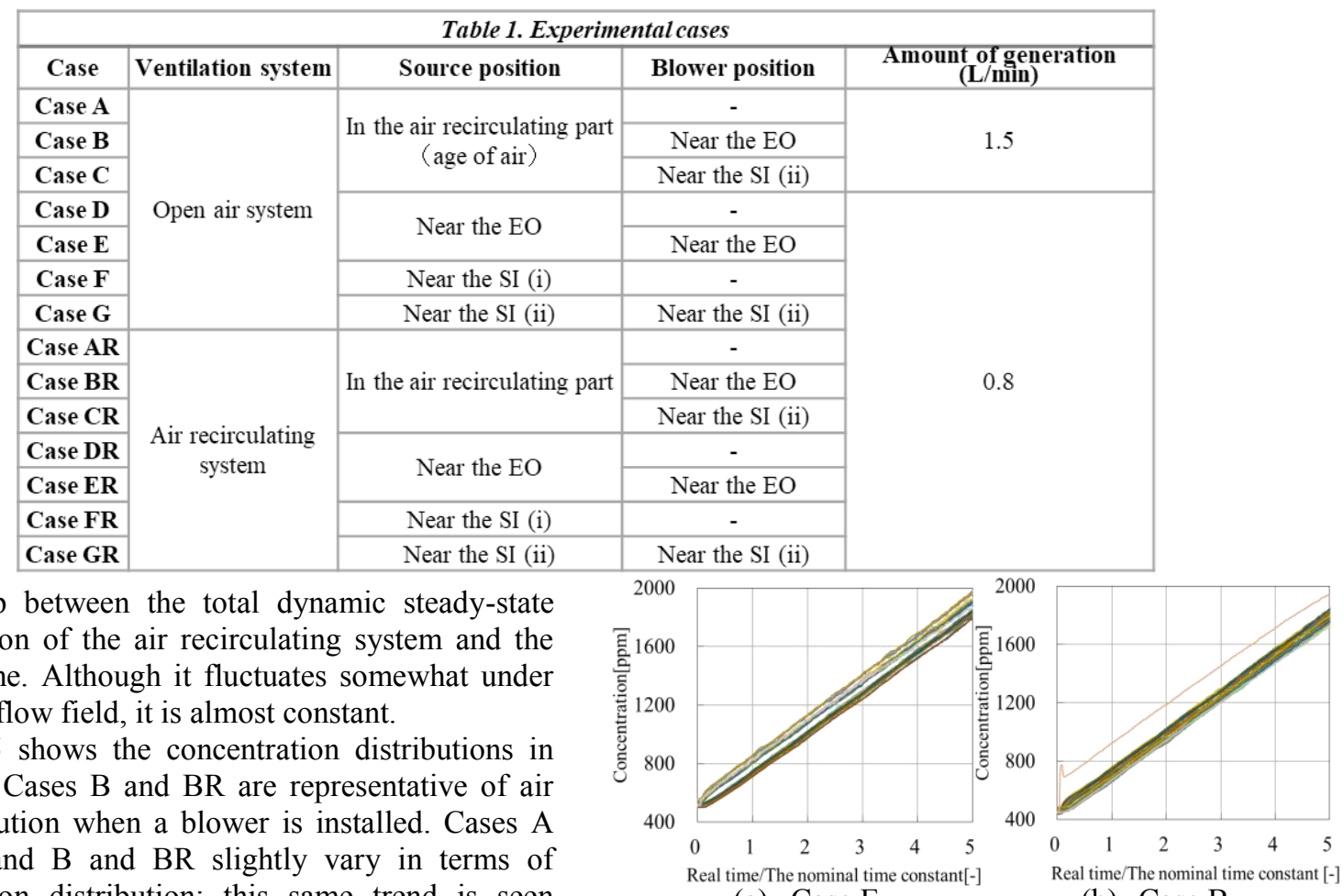

(a) Case $E_{R}$

(b) Case $B_{R}$ concentration distribution; this same trend is seen throughout because the concentration distribution is low on the SI side and high on the EO side. From this, it is confirmed that the concentration distribution, in the case of the air recirculating point source, is equivalent to the age of air in the open-air system.

In comparing point source Case D with Case AR + DR, and Case F with Case AR + FR, it is understood that a similar tendency is generally shown, that is the concentration is high in the vicinity of occurrence. It was found that the sum of the dynamic steady-state concentrations in a room with the air recirculating system corresponds to the steady-state concentration of the equivalent open-air system.

In the comparisons of Case E and Case BR + ER, and Case G and Case CR + GR, which occured inside the blower, the concentration distribution is found to be consistent between the open air system and the air recirculating system, resulting in a high concentration in the upper part of the occurrence because tracer gas is blown up to the top by the blower. In addition, cases DR and ER were compared to determine the influence of the presence or absence of a blower generated single room source. Case ER shows that the generation source was well stirred in the room compared to the situation in Case DR. Therefore, it can be confirmed that retention due to the density difference had not occurred.

Figure 6 shows the relationship between the consistency of concentration measured by the open-air and air recirculating systems. We calculated the RMS of the relationship between the data as an index study in Case D and Case AR + DR, where RMS = 0.12 and a slight variation is observed between data.

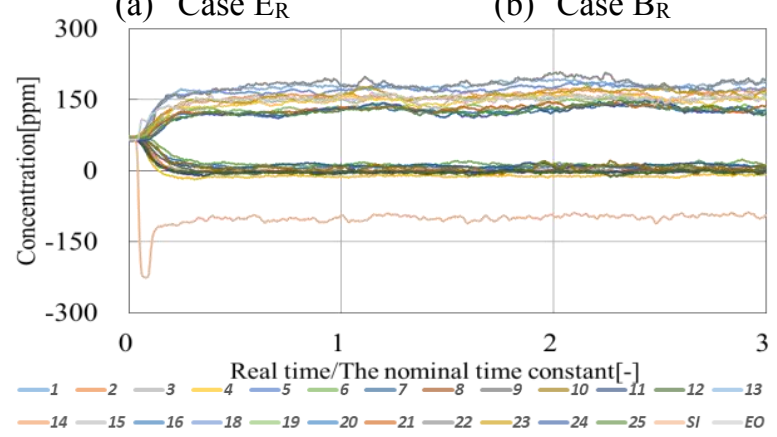

(c) Case $E_{R}+$ Case $B_{R}$

Figure. 4. The concentration transition of a dynamic steady-state concentration

However, Case E and Case BR + ER, in which blowers were installed at the same location, showed high correlation, with RMS $=0.05$. It is considered that the difference between the two correlations is due to the density difference between the $\mathrm{CO}_{2}$ gas used as a tracer gas and air. In point source generation, tracer gas stayed at the bottom of the occurrence and did not act as a strict passive scalar. In contrast, it is thought that the influence of the density difference became small when sufficient stirring was done using the blower.

On the other hand, Case F and Case AR + FR, which involved point source generators, are highly correlated, with $\mathrm{RMS}=0.04$. In cases F and FR had larger air flow at occurrence compared to cases D and DR. Therefore, it is thought that when tracer gas is stirred, the influence of the density difference is reduced.

As such, when the air flow at the point of occurrence in generating the point source was small, it was necessary to consider influence of the density difference between air and tracer gas. 

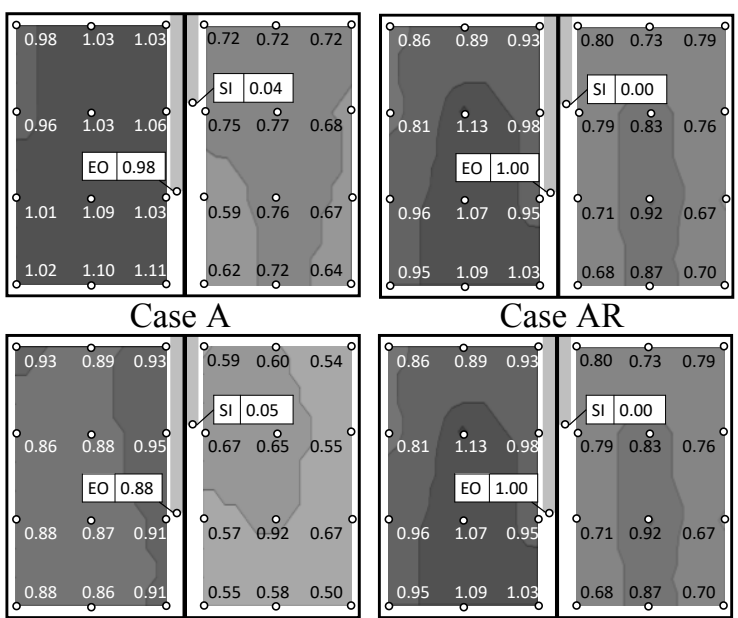

Case B

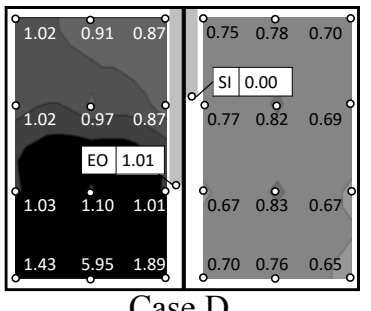

Case D

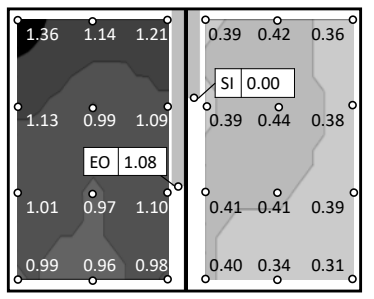

Case E

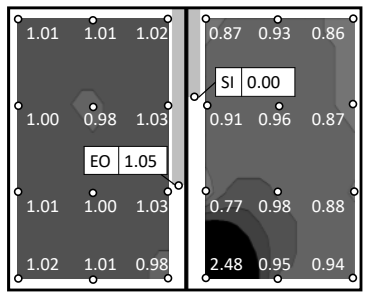

Case $\mathrm{F}$

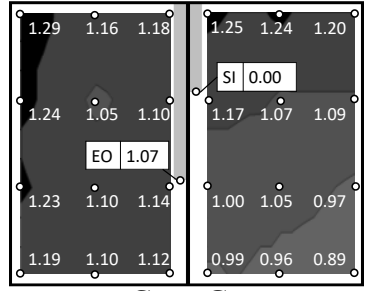

Case $\mathrm{G}$

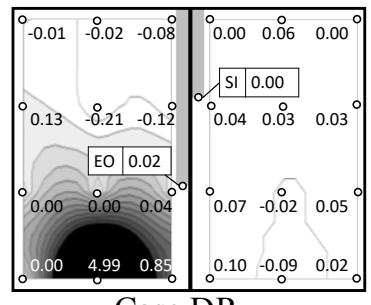

Case DR

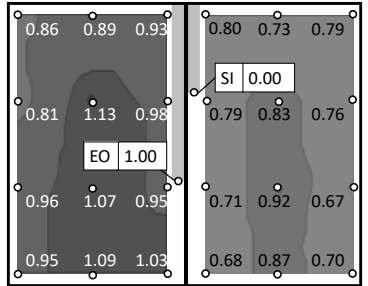

Case BR

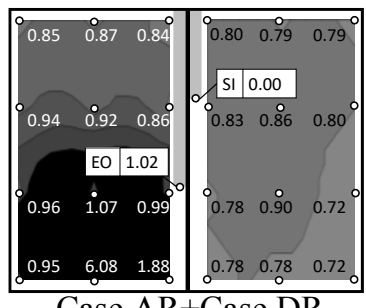

Case AR+Case DR

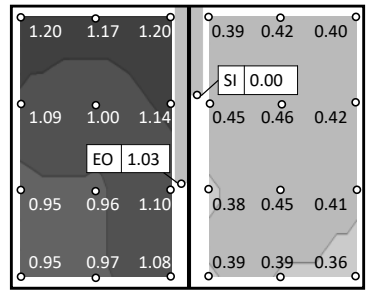

Case BR+Case ER

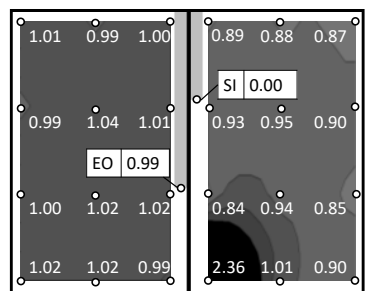

Case AR+Case FR

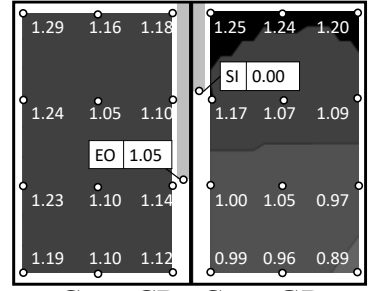

Case $\mathrm{CR}+$ Case GR

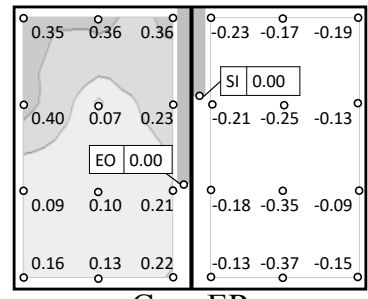

Case ER

Figure. 5. The measured concentration distribution
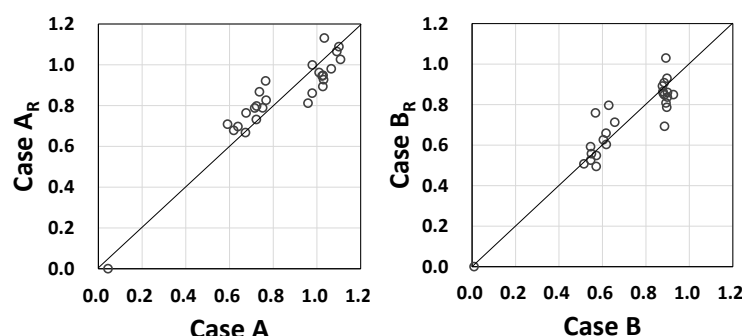

Case A : Case AR

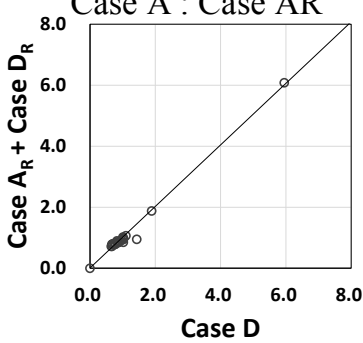

${ }_{1.4}$ Case B : Case BR

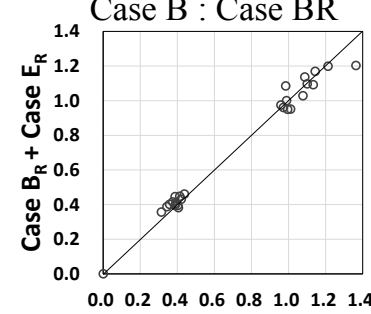

Case $\mathrm{E}$

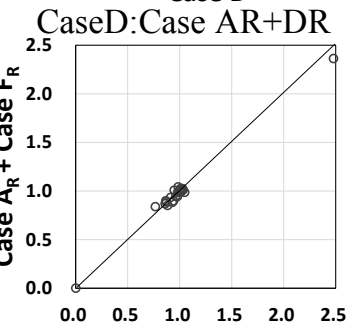

Case $F$

Case F:Case AR+FR

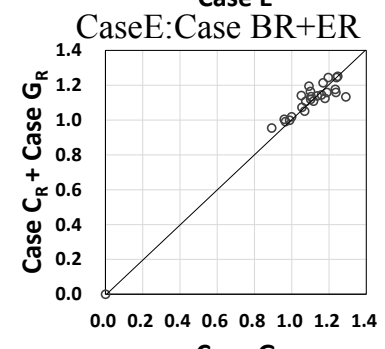

CaseG:Case CR+GR

Figure. 6. Consistency examination

\section{CONCLUSIONS}

We obtained the following findings:

1) The concentration distribution reversed sign when a positive source was generated in the air recirculating part corresponding to the equivalent age of air in a room with an open-air system.

2) The result of combining the dynamic steady-state concentrations in each room and the corresponding air recirculating part sources roughly corresponded to the steady-state concentration distribution in the equivalent open-air system.

3) When the tracer gas is sufficiently stirred at the location where the tracer gas is generated, the influence of the density difference can be ignored.

\section{Reference}

1 Kurabuchi T, Takeuchi J, Yoshino H, Toriumi Y, Lee S, Inoue $\mathrm{Y}$ and Miyajima $\mathrm{M}$. Tracer Gas Experimental Technique Based on Dynamic Steady State Concentration Applied to Air Recirculating Systems, Part 3. The Society of Heating, Air-Conditioning Sanitary Engineers of Japan, 2016, pp.21-24.

2 Kurabuchi T, Takeuchi J, Yoshino H, Toriumi Y and Miyajima M. Tracer Gas Test Method Using Dynamic Steady State Concentration Applied to Air Recirculating System with Leakage, Part 1. Environmental Engineering II. Architectural Institute of Japan, 2017, pp.737-740. 